\title{
The global solution and blow-up phenomena to a modified Novikov equation
}

\author{
Shaoyong Lai, , Haibo Yan and Nan Li
}

"Correspondence:

laishaoy@swufe.edu.cn

Department of Applied

Mathematics, Southwestern

University of Finance and

Economics, Chengdu, 610074,

China

\begin{abstract}
A modified Novikov equation with symmetric coefficients is investigated. Provided that the initial value $u_{0} \in H^{s}(R)\left(s>\frac{3}{2}\right),\left(1-\partial_{x}^{2}\right) u_{0}$ does not change sign and the solution $u$ itself belongs to $L^{1}(R)$, the existence and uniqueness of the global strong solutions to the equation are established in the space

$C\left([0, \infty) ; H^{s}(R)\right) \cap C^{1}\left([0, \infty) ; H^{s-1}(R)\right)$. A blow-up result to the development of singularities in finite time for the equation is acquired.
\end{abstract}

MSC: $35 \mathrm{G} 25 ; 35 \mathrm{~L} 05$

Keywords: global existence; strong solutions; blow-up result

\section{Introduction}

Many scholars have paid attention to the integrable equation

$$
u_{t}-u_{t x x}+4 u^{2} u_{x}=3 u u_{x} u_{x x}+u^{2} u_{x x x}
$$

which was derived by Novikov [1]. Well-posedness of the Novikov equation in the Sobolev spaces on the torus was first done by Tiglay in [2], and was completed on both the line and the circle by Himonas and Holliman in [3]. Its Hölder continuity properties were studied in Himonas and Holmes [4]. The periodic and the non-periodic Cauchy problem for Eq. (1) and continuity results for the data-to-solution map in the Sobolev spaces are discussed in Grayshan [5]. A matrix Lax pair for Eq. (1) is acquired in [6] and is shown to be related to a negative flow in the Sawada-Kotera hierarchy. The scattering theory is applied to find non-smooth explicit soliton solutions with multiple peaks for Eq. (1) in [7]. Sufficient conditions on the initial data to guarantee the formation of singularities in finite time for Eq. (1) are given in Jiang and Ni [8]. This multiple peak property is common with the Camassa-Holm and Degasperis-Procesi equations [9-11]. Mi and Mu [12] established many dynamic results for a modified Novikov equation with peak solution. It is shown in Ni and Zhou [13] that the Novikov equation associated with initial value has locally well-posedness in a Sobolev space $H^{s}$ with $s>\frac{3}{2}$ by using the abstract Kato theorem. Two results about the persistence properties of the strong solution for Eq. (1) are established in [13]. Using the Littlewood-Paley decomposition and nonhomogeneous Besov spaces, Yan et al. [14] proved the global existence and blow-up phenomena for the weakly dissipative Novikov equation. For other methods to handle the Novikov equation and the related partial differential equations, the reader is referred to [15-22] and the references therein.

(02014 Lai et al.; licensee Springer. This is an Open Access article distributed under the terms of the Creative Commons Attribution License (http://creativecommons.org/licenses/by/2.0), which permits unrestricted use, distribution, and reproduction in any medium, provided the original work is properly cited. 
Observing the coefficients of the Novikov equation (1), we see that the coefficient of $u^{2} u_{x}$ is equal to the coefficient of $u u_{x} u_{x x}$ plus the coefficient of $u^{2} u_{x x x}$. That is,

$$
4=3+1
$$

Indeed, this relationship among the coefficients plays important roles in the study of the essential dynamical properties of the Novikov model $[1,2,11-13]$. This motivates us to study the following equation:

$$
u_{t}-u_{t x x}+(a+b) u^{2} u_{x}=a u u_{x} u_{x x}+b u^{2} u_{x x x}
$$

where $a>0$ and $b>0$ are arbitrary constants. Clearly, letting $a=3$ and $b=1$, Eq. (2) becomes the Novikov equation (1). The essential difference between Eq. (2) and the Novikov equation (1) is that Eq. (2) does not conform with the following conservation law:

$$
\int_{R}\left(u^{2}+u_{x}^{2}\right) d x=\int_{R}\left(u_{0}^{2}+u_{0 x}^{2}\right) d x
$$

which results in the bounds of $\|u(t, \cdot)\|_{L^{\infty}(R)}$ for Eq. (1).

Making use of $u_{0} \in H^{s}(R), s>\frac{3}{2}$, the assumption that $\left(1-\partial_{x}^{2}\right) u_{0}$ does not change sign, and the assumption that the solution of Eq. (2) satisfies $u \in L^{1}(R)$, we prove the global existence theorem of Eq. (2) in the Sobolev space,

$$
u(t, x) \in C\left([0, \infty) ; H^{s}(R)\right) \cap C^{1}\left([0, \infty) ; H^{s-1}(R)\right) .
$$

The objective of this work is to investigate Eq. (2). Since $a>0$ and $b>0$ are arbitrary constants, we cannot obtain the boundedness of the solution $u$ for Eq. (2) although the initial data satisfy the sign condition. To overcome this, assuming that the solution itself satisfies $u \in L^{1}(R)$ and the initial data satisfy the sign condition, we adopt the methods used in Rodriguez-Blanco [16] to derive that $\left\|\frac{\partial u(t, x)}{\partial x}\right\|_{L^{\infty}(R)}$ possesses bounds for any time $t>0$. This leads us to establish the well-posedness of the global strong solutions to Eq. (2). Parts of the main results in $[17,18]$ are extended. In addition, we acquire a blow-up result to the development of singularities in finite time, which includes the blow-up result in [12].

The rest of this paper is organized as follows. Section 2 states the main results of this work. Section 3 proves the global existence result. The proof of a blow-up result is given in Section 4.

\section{Main results}

We let $L^{p}=L^{p}(R)(1 \leq p<+\infty)$ be the space of all measurable functions $h$ such that $\|h\|_{L^{p}}^{p}=\int_{R}|h(t, x)|^{p} d x<\infty$. We define $L^{\infty}=L^{\infty}(R)$ with the standard norm $\|h\|_{L^{\infty}}=$ $\inf _{m(e)=0} \sup _{x \in R \backslash e}|h(t, x)|$. For any real number $s$, we let $H^{s}=H^{s}(R)$ denote the Sobolev space with the norm defined by

$$
\|h\|_{H^{s}}=\left(\int_{R}\left(1+|\xi|^{2}\right)^{s}|\hat{h}(t, \xi)|^{2} d \xi\right)^{\frac{1}{2}}<\infty
$$

where $\hat{h}(t, \xi)=\int_{R} e^{-i x \xi} h(t, x) d x$. Here we note that the norms $\|\cdot\|_{L^{p}}^{p},\|\cdot\|_{L^{\infty}}$ and $\|\cdot\|_{H^{s}}$ depend on variable $t$. 
For $T>0$ and nonnegative number $s, C\left([0, T) ; H^{s}(R)\right)$ denotes the Frechet space of all continuous $H^{s}$-valued functions on $[0, T)$. We set $\Lambda=\left(1-\partial_{x}^{2}\right)^{\frac{1}{2}}$.

In order to study the existence of solutions for Eq. (2), we consider its Cauchy problem in the form

$$
\left\{\begin{array}{l}
u_{t}-u_{t x x}+(a+b) u^{2} u_{x}=a u u_{x} u_{x x}+b u^{2} u_{x x x} \\
u(0, x)=u_{0}(x)
\end{array}\right.
$$

which is equivalent to

$$
\left\{\begin{array}{l}
u_{t}+b u^{2} u_{x}=\Lambda^{-2}\left[\left(-a u^{2} u_{x}+\frac{a-6 b}{2}\left(u u_{x}^{2}\right)_{x}+\frac{2 b-a}{2} u_{x}^{3}\right],\right. \\
u(0, x)=u_{0}(x)
\end{array}\right.
$$

where $a>0$ and $b>0$ are arbitrary constants. Now we give the main results for problem (3).

Theorem 1 Assume that the solution of problem (3) satisfies $u(t, x) \in L^{1}(R)$ and let $u_{0}(x) \in$ $H^{s}, s>\frac{3}{2}$ and $\left(1-\partial_{x}^{2}\right) u_{0} \geq 0$ for all $x \in R$ (or equivalently $\left(1-\partial_{x}^{2}\right) u_{0} \leq 0$ for all $x \in R$ ). Then problem (3) has a unique solution satisfying

$$
u(t, x) \in C\left([0, \infty) ; H^{s}(R)\right) \cap C^{1}\left([0, \infty) ; H^{s-1}(R)\right) .
$$

Theorem 2 Assume that $u_{0}(x) \in H^{s}(R)$ with $s>\frac{3}{2}$. If $a=b$, then every solution of problem (3) exists globally in time. If $a>b$, then the solution blows up in finite time if and only if $u u_{x}$ becomes unbounded from below in finite time. If $a<b$, then the solution blows up in finite time if and only if $u u_{x}$ becomes unbounded from above in finite time.

\section{Global strong solutions}

For proving the global existence for problem (3), we cite the local well-posedness result presented in [18].

Lemma $3.1([18])$ Let $u_{0}(x) \in H^{s}(R)$ with $s>\frac{3}{2}$. Then the Cauchy problem (3) has a unique solution $u(t, x) \in C\left([0, T) ; H^{s}(R)\right) \cap C^{1}\left([0, T) ; H^{s-1}(R)\right)$ where $T>0$ depends on $\left\|u_{0}\right\|_{H^{s}(R)}$.

Assume $u_{0} \in H^{s}(R)$ with $s>\frac{3}{2}$. Then there exists a unique solution $u(t, x)$ to problem (3) and

$$
u(t, x) \in C\left([0, T) ; H^{s}(R)\right) \cap C^{1}\left([0, T) ; H^{s-1}(R)\right)
$$

with the maximal existence time $T>0$. First, we study the differential equation

$$
\left\{\begin{array}{l}
p_{t}=b u^{2}(t, p), \quad t \in[0, T) \\
p(0, x)=x
\end{array}\right.
$$

Lemma 3.2 Let $u_{0} \in H^{s}, s>3$ and let $T>0$ be the maximal existence time of the solution to problem (3). Then problem (5) has a unique solution $p \in C^{1}([0, T) \times R, R)$. Moreover, the map $p(t, \cdot)$ is an increasing diffeomorphism of $R$ with $p_{x}(t, x)>0$ for $(t, x) \in[0, T) \times R$. 
Proof From Lemma 3.1, we have $u \in C^{1}\left([0, T) ; H^{s-1}(R)\right)$ and $H^{s-1} \in C^{1}(R)$. Thus we conclude that both functions $u(t, x)$ and $u_{x}(t, x)$ are bounded, Lipschitz in space and $C^{1}$ in time. Using the existence and uniqueness theorem of ordinary differential equations derives that problem (5) has a unique solution $p \in C^{1}([0, T) \times R, R)$.

Differentiating Eq. (5) with respect to $x$ yields

$$
\left\{\begin{array}{l}
\frac{d}{d t} p_{x}=2 b u u_{x}(t, p) p_{x}, \quad t \in[0, T), b \neq 0 \\
p_{x}(0, x)=1
\end{array}\right.
$$

which leads to

$$
p_{x}(t, x)=\exp \left(\int_{0}^{t} 2 b u u_{x}(\tau, p(\tau, x)) d \tau\right)
$$

For every $T^{\prime}<T$, using the Sobolev imbedding theorem yields

$$
\sup _{(\tau, x) \in\left[0, T^{\prime}\right) \times R}\left|u u_{x}(\tau, x)\right|<\infty .
$$

It is inferred that there exists a constant $K_{0}>0$ such that $p_{x}(t, x) \geq e^{-K_{0} t}$ for $(t, x) \in$ $[0, T) \times R$. It completes the proof.

Lemma 3.3 Let $u_{0} \in H^{s}$ with $s>3$, and let $T>0$ be the maximal existence time of the problem (3). We have

$$
y(t, p(t, x)) p_{x}^{2}(t, x)=y_{0}(x) e^{-(a-4 b) \int_{0}^{t} u u_{x} d \tau}
$$

where $(t, x) \in[0, T) \times R$ and $y:=u-u_{x x}$.

Proof Using Eqs. (2) and (6)-(8), we have

$$
\begin{aligned}
\frac{d}{d t} & {\left[y(t, p(t, x)) p_{x}^{2}(t, x)\right] } \\
& =y_{t} p_{x}^{2}+2 y p_{x} p_{x t}+y_{x} p_{t} p_{x}^{2} \\
& =y_{t} p_{x}^{2}+4 b y u u_{x} p_{x}^{2}+b u^{2} y_{x} p_{x}^{2} \\
& =\left(u_{t}-u_{t x x}+a u u_{x}\left(u-u_{x x}\right)+b u^{2}\left(u_{x}-u_{x x x}\right)\right) p_{x}^{2}-a u u_{x} y p_{x}^{2}+4 b u u_{x} y p_{x}^{2} \\
& =\left(u_{t}-u_{t x x}+(a+b) u^{2} u_{x}-a u u_{x} u_{x x}-b u^{2} u_{x x x}\right) p_{x}^{2}-(a-4 b) u u_{x} y p_{x}^{2} \\
& =-(a-4 b) u u_{x} y p_{x}^{2} .
\end{aligned}
$$

Using $p_{x}(0, x)=1$ and solving the above equation, we complete the proof of the lemma.

Remark 1 From Lemma 3.3, we conclude that, if $u_{0}-u_{0 x x}=\left(1-\partial_{x}^{2}\right) u_{0} \geq 0$, then $(1-$ $\left.\partial_{x}^{2}\right) u(t, x) \geq 0$. Since the operator $\left(1-\partial_{x}^{2}\right)^{-1}$ preserves positivity, we get $u \geq 0$. Similarly, if $\left(1-\partial_{x}^{2}\right) u_{0} \leq 0$, we have $\left(1-\partial_{x}^{2}\right) u \leq 0$ and $u \leq 0$. 
Lemma 3.4 If $u_{0} \in H^{s}, s>\frac{3}{2}$, such that $\left(1-\partial_{x}^{2}\right) u_{0} \geq 0\left(\operatorname{or}\left(1-\partial_{x}^{2}\right) u_{0} \leq 0\right)$ and $\int_{R}|u| d x<\infty$, then there exists a constant $K>0$ such that the solution of problem (3) satisfies $\left\|u_{x}\right\|_{L^{\infty}} \leq K$.

Proof We will prove this lemma to assume $u_{0} \in H^{\infty}$ which results in $u \in H^{\infty}$ from Lemma 3.1. For $\left(1-\partial_{x}^{2}\right) u_{0} \geq 0$, from Lemma 3.3, we have $\left(1-\partial_{x}^{2}\right) u \geq 0$. Then $u \geq 0$ does not change sign. From the assumption $\int_{R}|u| d x<\infty$ one derives

$$
-u_{x}+\int_{-\infty}^{x} u d x=\int_{-\infty}^{x}\left(u-u_{x x}\right) d x \leq \int_{-\infty}^{\infty}\left(u-u_{x x}\right) d x=c
$$

where $c$ is a positive constant. Then

$$
-u_{x} \leq c-\int_{-\infty}^{x} u d x \leq c+\int_{-\infty}^{x} u d x \leq 2 c
$$

On the other hand, we have

$$
u_{x}+\int_{x}^{\infty} u d x=\int_{x}^{\infty}\left(u-u_{x x}\right) d x \leq \int_{-\infty}^{\infty}\left(u-u_{x x}\right) d x=c
$$

which results in

$$
u_{x} \leq c-\int_{x}^{\infty} u d x \leq c+\int_{x}^{\infty} u d x \leq 2 c .
$$

We conclude from Eqs. (11) and (13) that $\left\|u_{x}\right\|_{L^{\infty}} \leq K$. To complete the proof, we use a simple density argument [16]. Setting $u_{0}^{\varepsilon}=e^{\varepsilon \partial_{x}^{2}} u_{0}$, we have $u_{0}^{\varepsilon} \in H^{\infty}$ and $\left\|u_{x}^{\varepsilon}\right\|_{L^{\infty}} \leq$ $2 \int_{R}|u| d x<K$. Applying $\left\|u_{x}^{\varepsilon}-u_{x}\right\|_{L^{\infty}} \leq \sup _{[0, T]}\left\|u_{x}^{\varepsilon}-u_{x}\right\|_{H^{s}} \rightarrow 0$ when $\varepsilon \rightarrow 0$, we have $\left\|u_{x}\right\|_{L^{\infty}} \leq K$.

Using the first equation of system (3) one derives

$$
\frac{d}{d t} \int_{R}\left(u^{2}+u_{x}^{2}\right) d x+2(a-3 b) \int_{R} u u_{x}^{3} d x=0
$$

from which we have the conservation law

$$
\int_{R}\left(u^{2}+u_{x}^{2}\right) d x+2(a-3 b) \int_{0}^{t} \int_{R} u u_{x}^{3} d x=\int_{R}\left(u_{0}^{2}+u_{0 x}^{2}\right) d x
$$

Lemma 3.5 (Kato and Ponce [23]) If $r \geq 0$, then $H^{r} \cap L^{\infty}$ is an algebra. Moreover

$$
\|u v\|_{r} \leq c\left(\|u\|_{L^{\infty}}\|v\|_{r}+\|u\|_{r}\|v\|_{L^{\infty}}\right)
$$

where $c$ is a constant depending only on $r$.

Lemma 3.6 (Kato and Ponce [23]) Let $r>0$. If $u \in H^{r} \cap W^{1, \infty}$ and $v \in H^{r-1} \cap L^{\infty}$, then

$$
\left\|\left[\Lambda^{r}, u\right] v\right\|_{L^{2}} \leq c\left(\left\|\partial_{x} u\right\|_{L^{\infty}}\left\|\Lambda^{r-1} v\right\|_{L^{2}}+\left\|\Lambda^{r} u\right\|_{L^{2}}\|v\|_{L^{\infty}}\right)
$$


Lemma 3.7 Let $s>\frac{3}{2}$ and the function $u(t, x)$ is a solution of problem (3) and the initial data $u_{0}(x) \in H^{s}(R)$. Then the following results hold:

$$
\|u\|_{L^{\infty}} \leq\|u\|_{H^{1}} \leq\left\|u_{0}\right\|_{H^{1}(R)} e^{\frac{|a-3 b|}{2} \int_{0}^{t}\left\|u_{x}\right\|_{L^{\infty}(R)}^{2} d \tau} .
$$

For $q \in(0, s-1]$, there is a constant $c$ only depending on $a$ and $b$ such that

$$
\begin{aligned}
\int_{R}\left(\Lambda^{q+1} u\right)^{2} d x \leq & \int_{R}\left[\left(\Lambda^{q+1} u_{0}\right)^{2}\right] d x \\
& +c \int_{0}^{t}\|u\|_{H^{q+1}}^{2}\left(\left\|u_{x}\right\|_{L^{\infty}}\|u\|_{L^{\infty}}+\left\|u_{x}\right\|_{L^{\infty}}^{2}\right) d \tau .
\end{aligned}
$$

Proof Using $\left|2 u u_{x}\right| \leq\left(u^{2}+u_{x}^{2}\right)$, the Gronwall inequality and Eq. (14), one derives Eq. (15).

Using $\partial_{x}^{2}=-\Lambda^{2}+1$ and the Parseval equality gives rise to

$$
\int_{R} \Lambda^{q} u \Lambda^{q} \partial_{x}^{3}\left(u^{3}\right) d x=-3 \int_{R}\left(\Lambda^{q+1} u\right) \Lambda^{q+1}\left(u^{2} u_{x}\right) d x+3 \int_{R}\left(\Lambda^{q} u\right) \Lambda^{q}\left(u^{2} u_{x}\right) d x .
$$

For $q \in(0, s-1]$, applying $\left(\Lambda^{q} u\right) \Lambda^{q}$ to both sides of the first equation of system (3) and integrating with respect to $x$ by parts, we have the identity

$$
\begin{aligned}
\frac{1}{2} \frac{d}{d t} \int_{R}\left[\left(\Lambda^{q} u\right)^{2}+\left(\Lambda^{q} u_{x}\right)^{2}\right] d x= & -a \int_{R}\left(\Lambda^{q} u\right) \Lambda^{q}\left(u^{2} u_{x}\right) d x \\
& -b \int_{R}\left(\Lambda^{q+1} u\right) \Lambda^{q+1}\left(u^{2} u_{x}\right) d x-2 b \int_{R} \Lambda^{q} u \Lambda^{q} u_{x}^{3} d x \\
& +(a-6 b) \int_{R} \Lambda^{q} u \Lambda^{q}\left(u u_{x} u_{x x}\right) d x
\end{aligned}
$$

We will estimate the terms on the right-hand side of Eq. (17) separately. For the first term, by using the Cauchy-Schwartz inequality and Lemmas 3.5 and 3.6, we have

$$
\begin{aligned}
\left|\int_{R}\left(\Lambda^{q} u\right) \Lambda^{q}\left(u^{2} u_{x}\right) d x\right|= & \left|\int_{R}\left(\Lambda^{q} u\right)\left[\Lambda^{q}\left(u^{2} u_{x}\right)-u^{2} \Lambda^{q} u_{x}\right] d x+\int_{R}\left(\Lambda^{q} u\right) u^{2} \Lambda^{q} u_{x} d x\right| \\
\leq & c\|u\|_{H^{q}}\left(2\|u\|_{L^{\infty}}\left\|u_{x}\right\|_{L^{\infty}}\|u\|_{H^{q}}+\left\|u_{x}\right\|_{L^{\infty}}\|u\|_{L^{\infty}}\|u\|_{H^{q}}\right) \\
& +\|u\|_{L^{\infty}}\left\|u_{x}\right\|_{L^{\infty}}\left\|\Lambda^{q} u\right\|_{L^{2}}^{2} \\
\leq & c\|u\|_{H^{q}}^{2}\|u\|_{L^{\infty}}\left\|u_{x}\right\|_{L^{\infty}} .
\end{aligned}
$$

Using the above estimate to the second term yields

$$
\left|\int_{R}\left(\Lambda^{q+1} u\right) \Lambda^{q+1}\left(u^{2} u_{x}\right) d x\right| \leq c\|u\|_{H^{q+1}}^{2}\|u\|_{L^{\infty}}\left\|u_{x}\right\|_{L^{\infty}} .
$$

Using the Cauchy-Schwartz inequality and Lemma 3.5, we obtain

$$
\begin{aligned}
\left|\int_{R}\left(\Lambda^{q} u_{x}\right) \Lambda^{q}\left(u u_{x}^{2}\right) d x\right| & \leq\left\|\Lambda^{q} u_{x}\right\|_{L^{2}}\left\|\Lambda^{q}\left(u u_{x}^{2}\right)\right\|_{L^{2}} \\
& \leq c\|u\|_{H^{q+1}}\left(\|u\|_{L^{\infty}}\left\|u_{x}^{2}\right\|_{H^{q}}+\|u\|_{H^{q}}\left\|u_{x}^{2}\right\|_{L^{\infty}}\right) \\
& \leq c\|u\|_{H^{q+1}}^{2}\left(\|u\|_{L^{\infty}}\left\|u_{x}\right\|_{L^{\infty}}+\left\|u_{x}\right\|_{L^{\infty}}^{2}\right) .
\end{aligned}
$$


For the last term in Eq. (17), using $u\left(u_{x}^{2}\right)_{x}=\left(u u_{x}^{2}\right)_{x}-u_{x} u_{x}^{2}$ results in

$$
\begin{aligned}
& \left|\int_{R}\left(\Lambda^{q} u\right) \Lambda^{q}\left(u u_{x} u_{x x}\right) d x\right| \\
& \quad \leq \frac{1}{2}\left|\int_{R} \Lambda^{q} u_{x} \Lambda^{q}\left(u u_{x}^{2}\right) d x\right|+\frac{1}{2}\left|\int_{R} \Lambda^{q} u \Lambda^{q}\left[u_{x} u_{x}^{2}\right] d x\right| \\
& \quad=K_{1}+K_{2} .
\end{aligned}
$$

For $K_{1}$, it follows from Eq. (20) that

$$
K_{1} \leq c\|u\|_{H^{q+1}}^{2}\left(\|u\|_{L^{\infty}}\left\|u_{x}\right\|_{L^{\infty}}+\left\|u_{x}\right\|_{L^{\infty}}^{2}\right)
$$

For $K_{2}$, applying Lemma 3.5 derives

$$
\begin{aligned}
K_{2} & \leq c\|u\|_{H^{q}}\left\|u_{x} u_{x}^{2}\right\|_{H^{q}} \\
& \leq c\|u\|_{H^{q}}\left(\left\|u_{x}\right\|_{L^{\infty}}\left\|u_{x}^{2}\right\|_{H^{q}}+\left\|u_{x}\right\|_{H^{q}}\left\|u_{x}^{2}\right\|_{L^{\infty}}\right) \\
& \leq c\|u\|_{H^{q+1}}^{2}\left\|u_{x}\right\|_{L^{\infty}}^{2} .
\end{aligned}
$$

It follows from Eqs. (18)-(23) that there exists a constant $c$ such that

$$
\frac{1}{2} \frac{d}{d t} \int_{R}\left[\left(\Lambda^{q} u\right)^{2}+\left(\Lambda^{q} u_{x}\right)^{2}\right] d x \leq c\|u\|_{H^{q+1}}^{2}\left(\left\|u_{x}\right\|_{L^{\infty}}\|u\|_{L^{\infty}}+\left\|u_{x}\right\|_{L^{\infty}}^{2}\right) .
$$

Integrating both sides of the above inequality with respect to $t$ results in inequality (16).

Proof of Theorem 1 Using Eq. (16) with $q=s-1$, we obtain

$$
\|u\|_{H^{s}}^{2} \leq\left\|u_{0}\right\|_{H^{s}}^{2}+c \int_{0}^{t}\|u\|_{H^{s}}^{2}\left(\|u\|_{L^{\infty}}\left\|u_{x}\right\|_{L^{\infty}}+\left\|u_{x}\right\|_{L^{\infty}}^{2}\right) d \tau .
$$

Applying the Gronwall inequality, we get

$$
\|u\|_{H^{s}}^{2} \leq\left\|u_{0}\right\|_{H^{s}}^{2} e^{2 c \int_{0}^{t}\left(\|u\|_{L} \infty\left\|u_{x}\right\|_{\left.L^{\infty}+\left\|u_{x}\right\|_{L^{\infty}}^{2}\right) d \tau}\right.} .
$$

Using Eq. (15) and Lemma 3.4, we complete the proof of Theorem 1.

Remark 2 In fact, using $\left\|u_{x}\right\|_{L^{\infty}} \leq\|u\|_{H^{s}}$ with $s>\frac{3}{2}$, Eqs. (15) and (26), we derive that the solution of Eq. (2) in space $H^{s}(R)$ blows up in finite time if and only if $\left\|u_{x}\right\|_{L^{\infty}}=+\infty$.

\section{Proof of Theorem 2}

Multiplying Eq. (2) by $y=u-u_{x x}$ and integrating by parts, we get

$$
\begin{aligned}
\frac{1}{2} \frac{d}{d t} \int_{R} y^{2} d x & =\int_{R} y y_{t} d x \\
& =\int_{R} y\left(a u u_{x} u_{x x}+b u^{2} u_{x x x}-(a+b) u^{2} u_{x}\right) d x
\end{aligned}
$$




$$
\begin{aligned}
& =\int_{R} y\left(a u u_{x}(u-y)+b u^{2}\left(u_{x}-y_{x}\right)-(a+b) u^{2} u_{x}\right) d x \\
& =\int_{R} y\left(-a u u_{x} y-b u^{2} y_{x}\right) d x \\
& =(b-a) \int_{R} u u_{x} y^{2} d x .
\end{aligned}
$$

When $a=b$, from Eq. (27), we derive $\left\|u_{x}\right\|_{L^{\infty}}$ is bounded. From Lemma 3.7 and Remark 2, we see that problem (3) has a global solution in the space

$$
C\left([0, \infty) ; H^{s}(R)\right) \cap C^{1}\left([0, \infty) ; H^{s-1}(R)\right) .
$$

Assume that the solution $u=u\left(\cdot, u_{0}\right)$ of problem (3) blows up in finite time in the space $H^{s}(R)$ with $s>\frac{3}{2}$. If $b-a<0$, we assume that $u u_{x}$ is bounded from below on $[0, T) \times R$, i.e., there exists a constant $M>1$ such that

$$
(b-a) u u_{x}(t, x) \leq M \quad \text { on }[0, T) \times R .
$$

From Eq. (27), we get

$$
\|u\|_{H^{2}} \leq c\left\|u_{0}\right\|_{H^{2}} e^{M t},
$$

from which we derive that the $H^{2}$ norm of the solution to problem (3) does not blow up in finite time. From Remark 2, we know that this is impossible. Therefore, we have $\lim _{t \rightarrow T} \inf _{\left\{\inf _{x \in R} u u_{x}(t, x)\right\}=-\infty}$.

Similar to the above, we know that if $b-a>0$, the solution of problem (3) blows up if and only if $\lim _{t \rightarrow T} \inf \left\{\inf _{x \in R} u u_{x}(t, x)\right\}=\infty$.

\section{Competing interests}

The authors declare that they have no competing interests.

\section{Authors' contributions}

The article is a joint work of three authors who contributed equally to the final version of the paper. All authors read and approved the final manuscript.

\section{Acknowledgements}

This work is supported by both the Fundamental Research Funds for the Central Universities (JBK130401, JBK120504) and the Applied and Basic Project of Sichuan Province (2012JY0020).

Received: 14 August 2013 Accepted: 23 December 2013 Published: 15 Jan 2014

\section{References}

1. Novikov, V: Generalizations of the Camassa-Holm equation. J. Phys. A, Math. Theor. 42, 342002 (2009)

2. Tiglay, F: The periodic Cauchy problem for Novikov's equation. Int. Math. Res. Not. 20, 4633-4648 (2011)

3. Himonas, AA, Holliman, C: The Cauchy problem for the Novikov equation. Nonlinearity 25, 449-479 (2012)

4. Himonas, AA, Holmes, J: Hölder continuity of the solution map for the Novikov equation. J. Math. Phys. 54, 061501 (2013)

5. Grayshan, K: Peakon solutions of the Novikov equation and properties of the data-to-solution map. J. Math. Anal. Appl. 397, 515-521 (2013)

6. Hone, ANW, Wang, JP: Integrable peakon equations with cubic nonlinearity. J. Phys. A, Math. Theor. 41, 372002 (2009)

7. Hone, ANW, Lundmark, H, Szmigielski, J: Explicit multipeakon solutions of Novikov's cubically nonlinear integrable Camassa-Holm type equation. Dyn. Partial Differ. Equ. 6, 253-289 (2009)

8. Jiang, ZH, Ni, LD: Blow-up phenomenon for the integrable Novikov equation. J. Math. Anal. Appl. 385, 551-558 (2012)

9. Camassa, R, Holm, D: An integrable shallow water equation with peaked solitons. Phys. Rev. Lett. 71, 1661-1664 (1993)

10. Constantin, A, Lannes, D: The hydro-dynamical relevance of the Camassa-Holm and Degasperis-Procesi equations. Arch. Ration. Mech. Anal. 193, 165-186 (2009) 
11. Constantin, A, Escher, J: Wave breaking for nonlinear nonlocal shallow water equations. Acta Math. 181, 229-243 (1998)

12. $\mathrm{Mi}, \mathrm{YS}, \mathrm{Mu}, \mathrm{CL}$ : On the Cauchy problem for the modified Novikov equation with peakon solutions. J. Differ. Equ. 254 961-982 (2013)

13. Ni, L, Zhou, Y: Well-posedness and persistence properties for the Novikov equation. J. Differ. Equ. 250, 3002-3021 (2011)

14. Yan, W, Li, YS, Zhang, YM: Global existence and blow-up phenomena for the weakly dissipative Novikov equation. Nonlinear Anal. 75, 2464-2473 (2012)

15. Himonas, A, Misiolek, G, Ponce, G, Zhou, Y: Persistence properties and unique continuation of solutions of Camassa-Holm equation. Commun. Math. Phys. 271, 511-522 (2007)

16. Rodriguez-Blanco, G: On the Cauchy problem for the Camassa-Holm equation. Nonlinear Anal. 46, $309-327$ (2001)

17. Lai, SY, Li, N, Wu, YH: The existence of global strong and weak solutions for the Novikov equation. J. Math. Anal. Appl. 399, 682-691 (2013)

18. Lai, SY, Wu, M: The local strong and weak solutions to a generalized Novikov equation. Bound. Value Probl. 2013, Article ID 134 (2013)

19. Lai, SY, Wu, YH: The local well-posedness and existence of weak solutions for a generalized Camassa-Holm equation J. Differ. Equ. 248, 2038-2063 (2010)

20. Yan, W, Li, YS, Zhang, YM: The Cauchy problem for the integrable Novikov equation. J. Differ. Equ. 253, 298-318 (2012)

21. Zhao, L, Zhou, SG: Symbolic analysis and exact travelling wave solutions to a new modified Novikov equation. Appl. Math. Comput. 217, 590-598 (2010)

22. Coclite, GM, Karlsen, KH: On the well-posedness of the Degasperis-Procesi equation. J. Funct. Anal. 223, 60-91 (2006)

23. Kato, T, Ponce, G: Commutator estimates and the Euler and Navier-Stokes equations. Commun. Pure Appl. Math. 41, 891-907 (1998)

10.1186/1687-2770-2014-16

Cite this article as: Lai et al.: The global solution and blow-up phenomena to a modified Novikov equation. Boundary Value Problems 2014, 2014:16

\section{Submit your manuscript to a SpringerOpen ${ }^{\ominus}$ journal and benefit from:}

- Convenient online submission

- Rigorous peer review

- Immediate publication on acceptance

- Open access: articles freely available online

- High visibility within the field

- Retaining the copyright to your article 